\title{
Middle meningeal artery embolization for the management of chronic subdural hematoma
}

\author{
David Fiorella, ${ }^{1,2}$ Adam S Arthur ${ }^{3}$
}

- Additional material is

published online only. To view please visit the journal online (http://dx.doi.org/10.1136/ neurintsurg-2019-014730).

${ }^{1}$ Department of Neurosurgery, Stony Brook University, Stony Brook, New York, USA

${ }^{2}$ SUNY SB, Stony Brook, New York, USA

3Univ Tennessee Health

Sciences Center Department of Neurosurgery/Semmes-Murphey Clinic, Memphis, Tennessee, USA

\section{Correspondence to}

Dr Adam S Arthur, UT

Department of Neurosurgeryl

Semmes-Murphey Clinic,

Memphis TN 38120, USA;

aarthur@semmes-murphey.com

Received 8 January 2019

Revised 28 January 2019

Accepted 31 January 2019

Published Online First

23 February 2019

\begin{abstract}
Chronic subdural hematoma (CSDH) is one of the most common neurosurgical diagnoses in adults. The disease is fundamentally a disorder of the meningeal blood vessels, and options exist for the minimally invasive neuroendovascular management. We review the potential role for the endovascular management of CSDH within the context of a discussion of the epidemiology, pathophysiology, and conventional management of this disease.
\end{abstract}

\section{THE DISEASE}

Chronic subdural hematoma (cSDH) is defined in the present discussion as a subdural collection of blood with components that are hypodense or isodense to brain on CT imaging. While the duration of the process is often unclear at the time of the initial diagnosis, it is thought that the process develops over a period of 3 or more weeks. At the time of diagnosis, many patients ( 9\%) may have a superimposed component of acute subdural blood intermixed with the chronic components and a history of recent trauma. In these cases it is important to differentiate the process from a hyperacute subdural hematoma $(\mathrm{SDH})$ or an acute $\mathrm{SDH}$ in a patient who is anticoagulated, as the isolated acute traumatic $\mathrm{SDH}$ represents a different pathology altogether. ${ }^{1-3}$

\section{CSDH IS A CEREBROVASCULAR PATHOLOGY}

cSDHs have a tendency to persist and gradually increase in volume over time. The disease is thought to be related to a cycle of chronic inflammation and angiogenesis. An original hemorrhage forms and fibrinolysis ensues with liquefaction of the initial clot. The subsequent blood breakdown products stimulate inflammation and thickening of the inner dural layer (ie, 'dural border cells'). This process incites angiogenesis with an ingrowth of immature capillaries, which chronically leak blood. These microhemorrhages result in the progressive enlargement of the collection with increased fibrinolytic activity, inflammation and further angiogenesis, membrane formation, and vessel proliferation. The rate of accumulation of blood products outpaces physiological reabsorption and the collection gradually enlarges. Thus the entire basis for the pathology is the formation of leaky vascular membranes, which incite a positive feedback cycle of continued hemorrhage, inflammation, and angiogenesis. ${ }^{45}$

Treatment strategies have been aimed at interrupting this cycle and tipping this balance back in favor of hemorrhage reabsorption. Surgical therapies involve the irrigation and removal of the blood products, sometimes with partial resection of these vascular membranes. ${ }^{6}$ Investigational medical therapies have employed various strategies, which include reducing the rate of microhemorrhage from the membranes, changing the osmotic environment, or altering angiogenesis. ${ }^{7}$ Endovascular therapies are aimed at de-vascularizing these membranes. ${ }^{8-11}$

\section{THE SCOPE OF THE PROBLEM}

$\mathrm{cSDH}$ is an extremely common disease process which is increasing in frequency each year. ${ }^{12}$ With the aging population and the prevalent use of anticoagulation and antiplatelet medications, by 2030 , there will be more than 60000 new cases of SDH per year, making it the most common neurosurgical diagnosis in adults at that time (with some estimates as high as $17-20$ cases per 100000 persons in the USA per year). ${ }^{13}$ This is more than double the rate of aneurysmal subarachnoid hemorrhage in the USA today.

Studies on the outcomes of cSDH patients have verified that this is a disabling and deadly diagnosis. ${ }^{14}$ Making the diagnosis itself can be challenging clinically, as patients present with a myriad of different symptoms. These symptoms are often initially non-focal and insidiously progressive and may not instigate early neuroimaging. 91516 Patients often present with behavioral and cognitive decline, and in this age population these changes can be easily attributed to progressive dementia, particularly if a diagnosis of early dementia has been assigned before. In fact, cSDH represents a significant etiology of reversible dementia. Other common symptoms include chronic headache, speech impairment, gait instability, seizure, and ultimately focal neurological signs with hemiparesis.

The inhospital morbidity and mortality rates very widely, but have typically been reported to be relatively low, ranging between $2 \%$ and $5 \% .^{15} 17$ In contrast, the 6 month and 1 year mortality rates have been consistently estimated at 30\%, ${ }^{14} 1819$ indicating that, like hip fracture or ischemic stroke, cSDH represents a significant sentinel health event which portends a marked reduction in life span for patients when compared with age matched controls.

\section{CONVENTIONAL MANAGEMENT}

There are no established guidelines for the management of patients with cSDH. As such, management strategies vary widely between individual physicians and institutions, and this variability is reflected in the literature. ${ }^{13}{ }^{14}$ In general, conservative management is restricted to patients who are asymptomatic or have minor symptoms, with a cSDH measuring $<10 \mathrm{~mm}$ in greatest thickness 
and with $<5 \mathrm{~mm}$ of midline shift. For other patients with more severe symptoms or larger cSDH, surgical management is generally favored.

\section{Surgical management of cSDH}

The percentage of patients who undergo surgery versus conservative management varies widely in the literature, from a low of $30 \%$ to almost $90 \% .^{13}{ }^{15}$ Surgical approaches have traditionally included twist drill hole, burr hole, and craniotomy based evacuations. Although many small comparative studies exist comparing the various surgical management techniques to each other, we have not found any organized studies that we are aware of comparing surgical management with observation.

Outcomes after surgical removal have generally been reported to be favorable, however, one major challenge is the tendency of the $\mathrm{cSDH}$ to recur after the initial evacuation, often times with neurological deterioration and a requirement for urgent or emergent re-operation. Reported rates of recurrence vary widely, ranging from $2 \%$ to $37 \%$ in various observational single center series, with most estimates falling between $10 \%$ and 20\%.12 $141720-24$ A second major challenge with surgical intervention is the requirement for the complete reversal of antiplatelet and anticoagulant agents, which are concomitant medications in a larger and larger proportion of afflicted patients. This necessary preoperative intervention comes with its own set of potential ischemic complications in this cohort. ${ }^{25}$ Finally, most patients with cSDH are elderly and have major comorbidities which could potentially complicate cranial surgery, ${ }^{26} 27$ and as such, less invasive options are attractive for selected patients.

\section{Conservative management of $\mathrm{CSDH}$}

Success rates for the conservative management of cSDH also vary widely between studies. Many authors state that spontaneous resolution of symptomatic cSDH is an unusual phenomenon with some studies reporting rates as low as $5 \%$ or less. ${ }^{28-30}$ Several medical management strategies have been applied in cSDH patients, including steroids, platelet activating factor antagonists, and statins. ${ }^{31}$ One of the more recent and prospective trials of medical management with steroids reported that of 26 consecutive patients (average hematoma size $21.9 \mathrm{~mm}$ ) treated with dexamethasone, 15 $(57.6 \%)$ failed and required surgical bailout. ${ }^{32}$ Other older, typically retrospective, series have reported somewhat more optimistic results in selected cSDH patients treated with steroids, with failure rates ranging between $15 \%$ and $30 \% .{ }^{33-35}$ A recent small retrospective case control trial evaluating statins in cSDH reported that of 24 patients, deterioration requiring surgery occurred in 2 of $12(16.7 \%)$ patients treated with atorvastatin versus 7 of $12(58.3 \%)$ in a case control arm. ${ }^{36}$ In a prospective study of a platelet activating factor antagonist, it was reported that 13 of 24 (54\%) of the treatment group and 27 of 29 (93\%) of the control group failed conservative/ medical management and required surgery. ${ }^{37}$ In summary, when reviewing the literature for conservative and/or medical management, the available studies are vexingly small and poorly controlled, and the reported rates of treatment failure requiring surgical rescue vary widely between $15 \%$ and $90 \%$. As such, the natural history of the disease is challenging to ascertain.

In addition, observational or medical therapies require prolonged hospitalization with immobilization or reduced activity, numerous serial imaging studies, and the discontinuation of antiplatelet and anticoagulant medications for a protracted period of time. Thus while avoiding surgery may be beneficial in some patients, this strategy also has potentially detrimental aspects to it.

\section{Middle meningeal artery embolization}

The goal of middle meningeal artery (MMA) embolization is to devascularize the subdural membranes to a sufficient extent such that the balance is shifted from the continued leakage and accumulation of blood products towards reabsorption. MMA embolization has been employed as the sole therapy (figure 1) and as a preoperative or postoperative adjunct to surgical evacuation with the intention of reducing postoperative recurrence. The procedure has typically been achieved with polyvinyl alcohol (PVA) particle embolization. Several small case series have reported success with this approach. ${ }^{8-11} 2538-41$

To date, the most rigorous evaluation of this treatment strategy was reported by Ban et al, who described outcomes in 72 consecutive patients who underwent MMA embolization, as sole therapy (27) or prior to surgical evacuation (45). The outcomes in the MMA embolization group were compared with a historical control group of $469 \mathrm{cSDH}$ patients who were treated conventionally. ${ }^{25}$ They defined treatment failure as either surgical rescue (ie, an operation in patients originally managed conservatively or
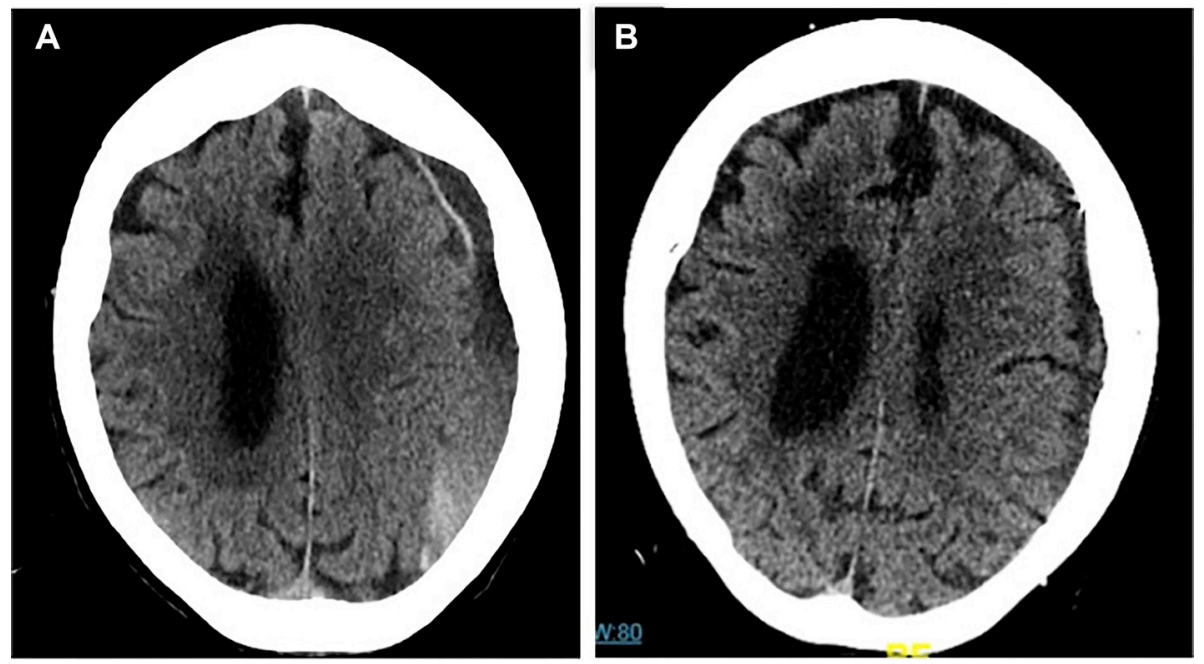

Figure 1 Adult patient presenting with a mixed density, chronic, left subdural hematoma with associated local mass effect (A). The patient underwent embolization of the middle meningeal artery branches with Onyx-18 (Medtronic, Irvine, California, USA). Follow-up CT (B) performed 12 weeks after the embolization demonstrates near complete resolution of the chronic subdural hematoma with resolution of the associated mass effect. 


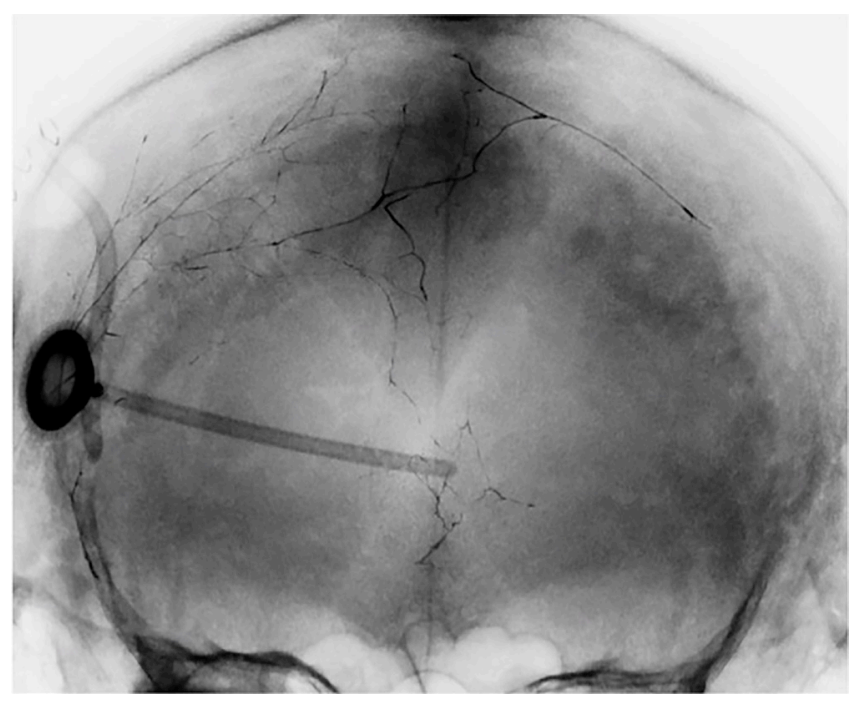

Figure 2 Posterior-anterior projection after Onyx embolization in a patient with recurrent chronic subdural hematoma following initial surgical evacuation. The Onyx can be easily visualized distributed within the distal branches of both middle meningeal arterial branches.

a re-operation after initial surgical management) or the incomplete resolution or re-accumulation of a subdural hematoma measuring $>10 \mathrm{~mm}$ at the 6 month follow-up CT. Of the 27 patients undergoing MMA embolization as sole therapy, there were no $(0 \%)$ treatment failures. Of the 45 patients undergoing MMA embolization prior to a planned surgical resection, there was only $1(2.2 \%)$ failure. When compared with case control data from the same institution, they reported failure in 56 of 67 $(83.6 \%)$ patients initially managed conservatively and in 73/402 (18.2\%) initially managed surgically. When reported as a composite, MMA embolization was successful in 71/72 (98.6\%) cases compared with a success rate of $340 / 469$ (72.5\%) in the control arm $(\mathrm{P}<0.001)$. Kim et al reported a smaller case control study comparing cSDH recurrence rates in surgical patients who underwent perioperative MMA embolization versus those who were treated conventionally. In this series of 20 patients undergoing MMA embolization, only 1 recurred (5\%). Of the 23 treated conventionally, $8(34.7 \%)$ recurred $(\mathrm{P}=0.024){ }^{40}$

Interestingly, in both of these case control studies, patients in the embolization arm were more likely to be on antiplatelet medications, and in the Kim et al study were more likely to have bilateral cSDH, in comparison with the conventional treatment arm. Conceivably, both of these variables would be expected to bias the results against the embolization cohort. Moreover, these data suggest that MMA embolization for sole treatment avoids the need for the disruption of anticoagulation and antiplatelet medications in these patients. In fact, in the Ban study, a significant percentage of the complications in the surgical arm of the case control group resulted either from ischemic events secondary to the reversal or discontinuation of antithrombotic medications or hemorrhagic events secondary to thrombocytopenia or the inadequate reversal of antithrombotic medications. ${ }^{25}$

Srivastan et al presented a meta-analysis of nine of the available MMA embolization case series and reported a markedly lower recurrence rate for $\mathrm{cSDH}$ after embolization compared with conventional management $(2.1 \%$ vs $27.7 \%$, OR 087, $95 \%$ CI 0.026 to $0.292, \mathrm{P}<0.001) .^{42}$

Thus while the available data are certainly preliminary, they are promising, and indicate a potential role for MMA embolization
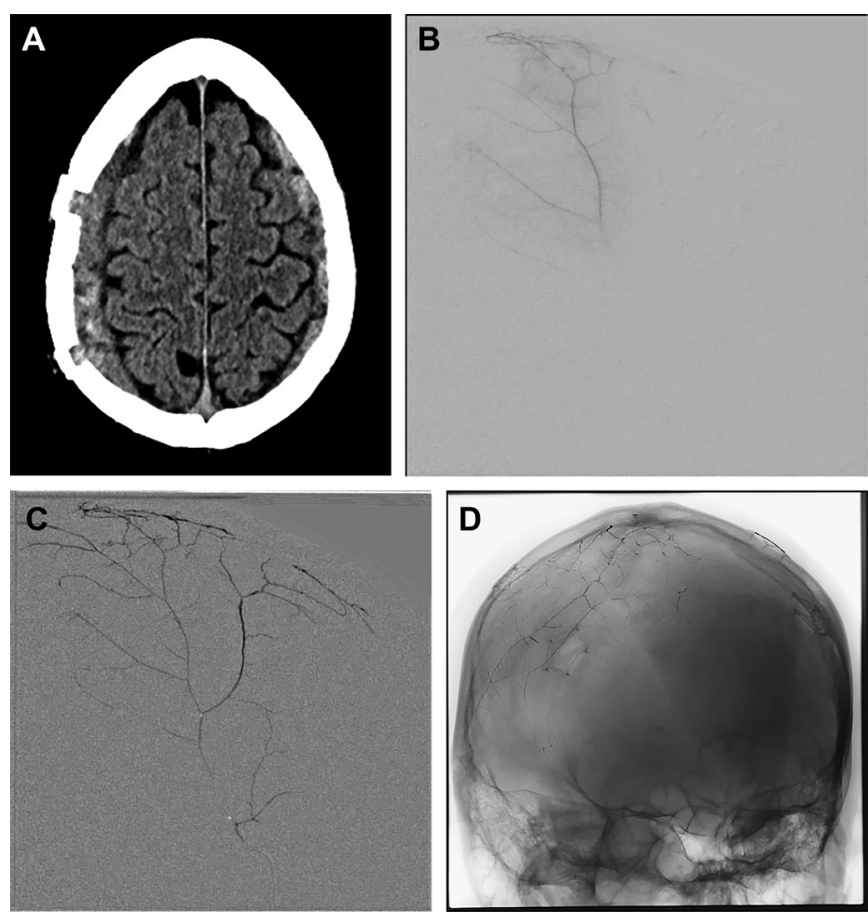

Figure 3 Adult patient with enlarging recurrent bilateral chronic subdural hematoma (cSDH) following initially successful burr hole craniotomy. CT of the head demonstrates bilateral mixed density cSDH collections (A). Superselective angiogram (B) from a catheter position within a frontal branch of the middle meningeal artery demonstrates the anatomy of the distal branches with a capillary blush of the dura. Blank fluoroscopic roadmap image in the same lateral projection demonstrates the Onyx-18 injection from this catheter position with extensive and distal casting of the targeted branch with backfilling of the adjacent regional meningeal branch vessels (C). Oblique radiograph (D) after embolization of the right sided meningeal branches demonstrating the conspicuity of the distribution of the liquid embolysate throughout the targeted meningeal branches over the right convexity.

for both the sole management and as a surgical adjunct for many patients with cSDH.

\section{TECHNICAL CONSIDERATIONS IN MIDDLE MENINGEAL ARTERY EMBOLIZATION}

While the vast majority of the existing case series were performed with PVA particulate embolysate, the potential advantages of liquid embolic agents for this purpose should be considered. First, particulates will only penetrate as distally as flow will allow. In older patient populations with $\mathrm{cSDH}$, these meningeal arteries are often times very small. As such, the microcatheter is not infrequently as large, or nearly as large, as the targeted vessel. For this reason, only a very small volume of PVA can be injected and the degree of distal penetration is limited. On the contrary, in a wedged catheter position, a liquid embolic agent can be infused into the distal vasculature and injected under pressure to achieve a casting of the subdural membranes and often times back reflux of adjacent meningeal branches (see online movie 1). This approach is very fast and technically straightforward. Second, PVA itself is not opaque. So during the injection, it is only visualized by watching the contrast carrier. The degree of distal penetration and the thoroughness of the embolization are difficult to discern. In addition, reflux into potentially eloquent branches can be difficult to observe. In comparison, liquid 
embolic agents are permanently opacified and their distribution is easily visualized (figures 2 and 3 ). This makes it much easier to gauge the distribution of the embolic agent and to visualize and control any potentially dangerous reflux. Finally, unlike PVA, liquid embolic agents are not resorbed and provide a durable and permanent embolization of the meningeal vasculature.

The advantages of PVA are that the existing data have been largely generated with this agent, it is inexpensive in comparison with liquid embolics, and the procedure can be performed under conscious sedation. ${ }^{910}$

\section{CONCLUSION}

The existing literature describing the natural history and surgical outcomes for patients with cSDH is composed largely of single center, retrospective, self-adjudicated case series. Most of the existing trials are neither prospective nor randomized and either compare different surgical techniques or are very small studies comparing a medical management strategy to observation. For this reason, it is difficult to ascertain a sense of the natural history of the group of cSDH patients who do not require emergency surgery. Superimposed on this background of uncertainty is the new minimally invasive technique of MMA embolization. While the existing studies suggest a robust treatment effect, they are relatively small and also not prospective or randomized. As such, an adequately sized, multicenter, prospective randomized controlled trial of MMA embolization both as sole therapy and as a surgical adjunct is needed. Such a trial will be critical in defining the safety and effectiveness (or lack thereof) of MMA embolization. Moreover, the data on conventional management will also be important in defining both the natural history and surgical outcomes for this disease state.

Contributors All named authors contributed substantially to the work described by actively participating in the study and the generation of the data, and providing editorial evaluation of the manuscript.

Funding The authors have not declared a specific grant for this research from any funding agency in the public, commercial, or not-for-profit sectors.

Competing interests ASA and DF are primary investigators for the STEM trial. Patient consent for publication Not required.

Provenance and peer review Commissioned; internally peer reviewed.

Author note This article was commisioned by the journal

\section{REFERENCES}

1 Sahyouni R, Goshtasbi K, Mahmoodi A, et al. Chronic subdural hematoma: a historical and clinical perspective. World Neurosurg 2017;108:948-53.

2 Sahyouni R, Goshtasbi K, Mahmoodi A, et al. Chronic subdural hematoma: a perspective on subdural membranes and dementia. World Neurosurg 2017; 108:954-8.

3 Sahyouni R, Mahboubi H, Tran P, et al. Membranectomy in chronic subdural hematoma: meta-analysis. World Neurosurg 2017;104:418-29.

4 Ito H, Yamamoto S, Komai T, et al. Role of local hyperfibrinolysis in the etiology of chronic subdural hematoma. J Neurosurg 1976;45:26-31.

5 Edlmann E, Giorgi-Coll S, Whitfield PC, et al. Pathophysiology of chronic subdural haematoma: inflammation, angiogenesis and implications for pharmacotherapy. J Neuroinflammation 2017:14:108.

6 Markwalder TM. The course of chronic subdural hematomas after burr-hole craniostomy with and without closed-system drainage. Neurosurg Clin N Am 2000;11:541-6.

7 Sun TF, Boet R, Poon WS. Non-surgical primary treatment of chronic subdural haematoma: preliminary results of using dexamethasone. Br J Neurosurg 2005:19:327-33.

8 Link TW, Boddu S, Marcus J, et al. Middle meningeal artery embolization as treatment for chronic subdural hematoma: a case series. Oper Neurosurg 2018;14:556-62.

9 Link TW, Boddu S, Paine SM, et al. Middle meningeal artery embolization for chronic subdural hematoma: a series of 60 cases. Neurosurgery 2018;121.

10 Link TW, Rapoport BI, Paine SM, et al. Middle meningeal artery embolization for chronic subdural hematoma: Endovascular technique and radiographic findings. Interv Neuroradiol 2018;24:455-62.
11 Link TW, Schwarz JT, Paine SM, et al. Middle meningeal artery embolization for recurrent chronic subdural hematoma: a case series. World Neurosurg 2018;118:e570-4.

12 Ducruet AF, Grobelny BT, Zacharia BE, et al. The surgical management of chronic subdural hematoma. Neurosurg Rev 2012;35:155-69. Discussion 69.

13 Balser D, Farooq S, Mehmood T, et al. Actual and projected incidence rates for chronic subdural hematomas in United States Veterans Administration and civilian populations. J Neurosurg 2015;123:1209-15.

14 Miranda LB, Braxton E, Hobbs J, et al. Chronic subdural hematoma in the elderly: not a benign disease. J Neurosurg 2011;114:72-6.

15 Ramachandran R, Hegde T. Chronic subdural hematomas-causes of morbidity and mortality. Surg Neurol 2007;67:367-72.

16 Cameron MM. Chronic subdural haematoma: a review of 114 cases. J Neurol Neurosurg Psychiatry 1978:41:834-9.

17 Weigel R, Schmiedek P, Krauss JK. Outcome of contemporary surgery for chronic subdural haematoma: evidence based review. J Neurol Neurosurg Psychiatry 2003;74:937-43.

18 Dumont TM, Rughani Al, Goeckes T, et al. Chronic subdural hematoma: a sentinel health event. World Neurosurg 2013;80:889-92.

19 Jones S, Kafetz K. A prospective study of chronic subdural haematomas in elderly patients. Age Ageing 1999;28:519-21.

20 Almenawer SA, Farrokhyar F, Hong C, et al. Chronic subdural hematoma management: a systematic review and meta-analysis of 34,829 patients. Ann Surg 2014;259:449-57.

21 Ivamoto HS, Lemos HP, Atallah AN. Surgical treatments for chronic subdural hematomas: a comprehensive systematic review. World Neurosurg 2016;86:399-418.

22 Liu W, Bakker NA, Groen RJ. Chronic subdural hematoma: a systematic review and meta-analysis of surgical procedures. J Neurosurg 2014;121:665-73.

23 Xu CS, Lu M, Liu LY, et al. Chronic subdural hematoma management: clarifying the definitions of outcome measures to better understand treatment efficacy - a systematic review and meta-analysis. Eur Rev Med Pharmacol Sci 2017;21:809-18.

24 Gernsback J, Kolcun JP, Jagid J. To drain or two drains: recurrences in chronic subdural hematomas. World Neurosurg 2016;95:447-50.

25 Ban SP, Hwang G, Byoun HS, et al. Middle meningeal artery embolization for chronic subdural hematoma. Radiology 2018;286:992-9.

26 Toi H, Kinoshita K, Hirai S, et al. Present epidemiology of chronic subdural hematoma in Japan: analysis of 63,358 cases recorded in a national administrative database. J Neurosurg 2018;128:222-8.

27 Uno M, Toi H, Hirai S. Chronic subdural hematoma in elderly patients: is this disease benign? Neurol Med Chir 2017;57:402-9.

28 Gjerris F, Schmidt K. Chronic subdural hematoma. Surgery or mannitol treatment. J Neurosurg 1974;40:639-42.

29 Kim HC, Ko JH, Yoo DS, et al. Spontaneous resolution of chronic subdural hematoma: close observation as a treatment strategy. J Korean Neurosurg Soc 2016;59:628-36.

30 Naganuma H, Fukamachi A, Kawakami M, et al. Spontaneous resolution of chronic subdural hematomas. Neurosurgery 1986;19:794-8.

31 Soleman J, Nocera F, Mariani L. The conservative and pharmacological management of chronic subdural haematoma. Swiss Med Wkly 2017;147:w14398.

32 Thotakura AK, Marabathina NR. Nonsurgical treatment of chronic subdural hematoma with steroids. World Neurosurg 2015;84:1968-72.

33 Pichert G, Henn V. [Conservative therapy of chronic subdural hematomas]. Schweiz Med Wochenschr 1987;117:1856-62.

34 Bender MB, Christoff N. Nonsurgical treatment of subdural hematomas. Arch Neurol 1974;31:73-9.

35 Delgado-López PD, Martín-Velasco V, Castilla-Díez JM, et al. Dexamethasone treatment in chronic subdural haematoma. Neurocirugia 2009;20:346-59.

36 Chan DY, Chan DT, Sun TF, et al. The use of atorvastatin for chronic subdural haematoma: a retrospective cohort comparison study. Br J Neurosurg 2017;31:72-7.

37 Hirashima Y, Kurimoto M, Nagai S, et al. Effect of platelet-activating factor receptor antagonist, etizolam, on resolution of chronic subdural hematoma-a prospective study to investigate use as conservative therapy. Neurol Med Chir 2005;45:621-6. Discussion 26

38 Hashimoto T, Ohashi T, Watanabe D, et al. Usefulness of embolization of the middle meningeal artery for refractory chronic subdural hematomas. Surg Neurol Int 2013;4:104

39 Kang J, Whang K, Hong SK, et al. Middle meningeal artery embolization in recurrent chronic subdural hematoma combined with arachnoid cyst. Korean J Neurotrauma 2015:11:187-90.

40 Kim E. Embolization therapy for refractory hemorrhage in patients with chronic subdural hematomas. World Neurosurg 2017:101:520-7.

41 Matsumoto $\mathrm{H}$, Hanayama $\mathrm{H}, \mathrm{Okada} \mathrm{T}$, et al. Which surgical procedure is effective for refractory chronic subdural hematoma? Analysis of our surgical procedures and literature review. J Clin Neurosci 2018;49:40-7.

42 Srivatsan A, Mohanty A, Nascimento FA, et al. Middle meningeal artery embolization for chronic subdural hematoma: meta-analysis and systematic review. World Neurosurg 2019;122:613-9. 